\title{
Midiendo el impacto del valor compartido: el caso de negocios
}

\author{
Mauricio Vladimir Umaña Ramírez \\ Doctor en Competitividad Empresarial y Desarrollo Económico \\ Docente investigador, Facultad de Ciencias Empresariales \\ Universidad Católica de El Salvador, El Salvador \\ Email: mauricio.umana@catolica.edu.sv \\ ORCID: https://orcid.org/0000-0002-0733-5183
}

Fecha de recepción: $07-12-2019$

Fecha de aceptación: $22-02-2020$

\section{Resumen}

El valor compartido es un concepto que, desde sus inicios en 2011, ha atraído el interés de académicos, gobiernos, empresarios y tomadores de decisión en todos los ámbitos. Tradicionalmente, la creación de valor ha sido confundida con la sostenibilidad, la responsabilidad social empresarial, entre otros; pero existe una línea que une todos los conceptos, a través de la creación de un valor social y un valor económico, generado simultáneamente.

Dentro de este estudio exploratorio, se creó el primer caso de negocios sobre cómo medir este valor, con el propósito de representar la creación de valor en variables de beneficio para la sociedad, tales como: salud y habilidades de los empleados; seguridad, impacto ambiental, uso del agua, uso de la energía, creación de nuevas microempresas, entre otras.

Los resultados de la investigación brindan la pauta para seguir midiendo más casos; ofreciendo una propuesta novedosa a nivel nacional e internacional, sobre cómo medir el valor compartido, y cómo la inversión social puede generar un retorno a la empresa.

Palabras clave: Valor social, valor económico, valor compartido, competitividad.

\begin{abstract}
Shared value is a concept that, since its inception in 2011, has attracted the interest of academics, governments, businesspersons and decision makers in all areas. Traditionally, value creation has been confused with sustainability, corporate social responsibility, among others; but there is a line that unites all the concepts, through the creation of a social value and an economic value, generated simultaneously.

Within this exploratory study, the first business case on how to measure this value was created, with the purpose to represent the creation of value in variables of benefit to society, such as: health and skills of employees; safety, environmental impact, use of water, use of energy, creation of new micro-businesses, among others.

The results of the investigation provide the guideline to continue measuring more cases; offering a novel proposal at national and international level, on how to measure shared value, and how social investment can generate a return to the company.
\end{abstract}

Key words: Social value, economic value, shared value, competitiveness. 


\section{Introducción}

En los tiempos actuales, uno de los principales problemas sobre la creación de valor social y valor económico en Latinoamérica es que muchas empresas continúan invirtiendo en actividades que están desconectadas de la estrategia de la empresa (Porter, 2002). Mientras muchas industrias sigan invirtiendo de esta forma, se están desperdiciando grandes oportunidades para recoger los frutos del valor (Porter et al. 2009).

Prahalad y Hart (2002) han aclarado que existe una fortuna en la base de la pirámide económica; pero para las empresas ha sido difícil encontrar ese valor. En el trabajo de Hart (2010) se puede ver claramente la necesidad de cambiar el modo tradicional del capitalismo, el cual se encuentra en una encrucijada. Probablemente este panorama refleja la gran coincidencia en la literatura con el concepto de valor compartido dado por Porter y Kramer (2011) en la nueva forma de hacer negocios.

Para Porter (2009), la ventaja competitiva de la empresa ha de ser sostenible, en la medida que las empresas sigan invirtiendo el valor en áreas que no mejoran completamente la productividad o las condiciones del mercado, o de los productos que se crean y venden. Esta sostenibilidad se vuelve prácticamente imposible, de allí que el vínculo entre la ventaja competitiva y la responsabilidad social existe (Porter y Kramer, 2006).

\section{Conceptualización}

Para Vidal (2011), la empresa crea valor compartido cuando su actuación genera un beneficio significativo para la sociedad, que también es de valor para el negocio. Estas pueden crear valor compartido por tres vías distintas, que se complementan constituyendo un círculo virtuoso: concebir nuevos productos y mercados; redefinir la productividad en la cadena de valor y construir clústeres locales donde actúa. En resumen, valor compartido implica innovación.

Para esta investigación, se define valor compartido como: "políticas y prácticas de empresa que realzan la competitividad de la compañía, al mismo tiempo que progresan los colaboradores y la comunidad en donde se vende y opera" (Porter y Kramer, 2011).

Vidal (2011) recomienda que los gestores de empresas tomen decisiones guiados por el principio del valor compartido, que significa la creación de valor económico de tal manera que, a la vez, facilite satisfacer las necesidades y aspiraciones de la sociedad. Valor compartido no es filantropía, es la nueva forma duradera de alcanzar el éxito económico.

De lo anterior, se desprende que un primer paso para las empresas, universidades e instituciones para la colaboración, es pensar en co-crear valor simultáneamente con los otros actores del entorno; no trabajar aisladamente. De lo contrario, las oportunidades de crear valor en la sociedad por las empresas y otras instituciones se pierden. 
Basado en Umaña (2019), el valor se puede perder si no se invierte el dinero en aquellas apuestas productivas que pueden desatar una ola de innovación y crecimiento en las empresas. A esto se le llama una especie de dinero inteligente, en la inversión en emprendimientos y en los primeros análisis. Umaña (2013) plantea que el mundo necesita que las empresas inviertan de forma inteligente su dinero para contribuir a la sostenibilidad, en el trabajo sobre la ecuación de la sostenibilidad.

A continuación, se crea toda una tipología del avance de la responsabilidad social de la empresa hasta la creación de valor. Se plantea que el avance de la literatura ha pasado por cinco etapas: filantropía, responsabilidad social empresarial voluntaria, respuesta de responsabilidad social empresarial, responsabilidad social empresarial estratégica y valor compartido; siendo esta última etapa donde se encuentra los vacíos y la confluencia de intereses, que las otras fases no pueden cubrir. Por ejemplo, la desconexión de la estrategia que se le acusa a la filantropía, como el defecto de actuar voluntario de la responsabilidad social empresarial, o la forma reactiva a los problemas sociales de la respuesta de responsabilidad social empresarial.

\section{Conceptos y evolución de la responsabilidad} social empresarial

Las discusiones sobre la importancia de la responsabilidad social empresarial (RSE) ${ }^{1}$ se han desarrollado alrededor de 80 años (Ballantine 1932, Berle 1931). Este análisis sugiere diferen- cias sustanciales en la forma de actuar de la corporación: desde la filantropía corporativa, RSE voluntaria, respuesta RSE y RSE estratégica.

\section{Filantropía corporativa}

En esta etapa, la filantropía se convierte en un componente voluntario de la compañía y consiste de una contribución voluntaria de recursos privados para propósitos públicos (Salomón, 1992). La filantropía es por definición voluntaria; por ende, ha recibido muchas críticas por sus esfuerzos desconectados de la estrategia (Porter y Kramer, 2002).

Ha habido enemigos de la RSE (Friedman, 1970), también ha habido abogados de la misma (Samuelson, 1971). Wang, Choi y Li (2008) concluyen que la relación entre filantropía corporativa y desempeño financiero es mejor captada por una forma de $\mathrm{U}$ invertida. De este análisis, se concluye que la filantropía es una etapa de la responsabilidad social de las empresas, pero está desconectada de la estrategia empresarial.

\section{Responsabilidad social empresarial voluntaria}

La filantropía corporativa evoluciona en la literatura hacia la RSE voluntaria, la cual refleja un compromiso continuo de las empresas para comportarse éticamente, y contribuir al desarrollo económico de sus empleados y familias, así como a la comunidad y la sociedad en general (Holme y Watts, 2000; Hediger, 2010).

Jamali y Mirshak (2007) han destacado la estrategia de estos temas en las economías en

1. Dentro del documento, el autor hará referencia a este término mediante su acrónimo. 
desarrollo y la falta de estudios empíricos sobre RSE, que desafortunadamente ha sido solo un proceso voluntario. A partir de este análisis, se propone que la RSE voluntaria es una segunda etapa de la RSE, desconectada de la estrategia empresarial.

\section{Respuesta responsabilidad social empresarial}

La RSE voluntaria evoluciona hacia la respues-

ta $\mathrm{RSE}$, reflejando una reacción de la empresa como un accionar corporativo sobre las consecuencias en sus accionistas (Freeman, 1984).

Porter y Kramer (2006) definen esta etapa como "buena ciudadanía corporativa y mitigar daños de las actividades de la empresa en la cadena de valor". Basado en lo anterior, se puede plantear que esta etapa sigue siendo reactiva y, por ende, sigue desconectada de la estrategia.

\section{Responsabilidad social empresarial estratégica}

La RSE estratégica representa una táctica empresarial integrada en todas las actividades de la cadena de valor de la empresa, observando cada decisión del negocio para contribuir simultáneamente a maximizar el valor a largo plazo para los accionistas; y los beneficios para la sociedad y el medio ambiente. Muchos autores han reflexionado sobre la ventaja competitiva de la RSE (Drucker, 1984; McWilliams y Siegel, 2001).

Uno de los primeros trabajos basados en la necesidad competitiva de la empresa y su valor social, ambiental y de negocios es el de Hart (1995), donde el desempeño social corporati- vo puede constituir una fuente de ventaja competitiva, y por otro lado, el de Russo y Fouts (1997), que es uno de los estudios empíricos pioneros. McElhaney (2009) proporciona una excelente definición de RSE estratégica: "una estrategia empresarial que se integra con los objetivos y las competencias centrales de la empresa y, desde el principio, está diseñada para crear valor comercial y un cambio social positivo; y está integrado en la cultura empresarial y en las operaciones diarias".

Finalmente, McWilliams, Siegel y Wright (2006) describieron la teoría de Hart (19952011) como la que permite a la empresa, desde la RSE, contribuir estratégicamente a una ventaja competitiva sostenible. Se propone que, en esta etapa, la empresa pueda contribuir en alto nivel a la creación de valor, y probablemente esta no es la etapa de maximización del valor.

\section{Valor compartido}

La forma más sofisticada de crear ese valor social desde la actividad empresarial se da a través del valor de negocio, que es invertido de forma inteligente para generar por cada dólar invertido, más ganancias y capacidades para la sociedad para el largo plazo.

En esta investigación se propone un modelo de valor compartido que realza la competitividad de la compañía (Porter y Kramer, 2011); por ejemplo, invirtiendo en "habilidades, formación y competencias" para los empleados. Pero esta inversión se va a traducir en grandes ahorros de productividad, en mejoras de manejo de inven- 
tarios y de calidad para la empresa. Este es el propósito del nuevo modelo de Capitalismo.

Es importante destacar que, en El Salvador, los proyectos de formación en "habilidades y competencias", salud de los empleados, inversión en escuelas, programas de alianzas con instituciones de la sociedad civil y el Estado, pueden reflejar fácilmente mejoras en la continuidad del negocio; ya que, desde la seguridad del trabajador y mejoras de su productividad, se le garantizan a él y a su familia, condiciones sociales de trabajo que son muy difíciles de lograr en el país.

La investigación trata de contestar las preguntas: ¿Existe un caso de negocio que pueda guiar a más investigaciones en Latinoamérica y El Salvador sobre el valor compartido? ¿Existe alguna relación entre la creación de valor compartido y el retorno de estas inversiones?

La primera pregunta puede indicar los próximos pasos que debe impulsar la academia y las empresas, sobre los cuales se puede acelerar más investigación. Por su parte, la segunda pregunta puede indicar el modelo de incremento de inversiones en valor compartido, para crear simultáneamente valor social, valor ambiental y valor económico.

Con base en lo analizado anteriormente, se propone la siguiente hipótesis: La creación valor compartido puede llevar a las empresas a maximizar el valor invertido en valor social, valor ambiental y convertirlo en un mayor valor económico.

\section{Metodología}

La investigación fue de tipo exploratoria y descriptiva, a través del estudio de caso (Zainal, 2007); de tal manera que los resultados fueron indicativos de cómo se comportan los casos en empresas grandes de más de 100 empleados. Este es un primer modelo que refleja datos estratégicos de al menos tres años de resultados consecutivos.

Este primer caso de estudio ejemplifica el comportamiento de la meta matriz, generada para captar la información de la empresa. Esta se circunscribe en Ciudad Arce, departamento de La Libertad, El Salvador. El lugar representa un excelente laboratorio, porque la empresa dota de actividad económica a toda esa zona, ya que no hay ninguna otra en más de $7 \mathrm{kms}^{2}$ a la redonda. Claro está, la creación de valor en la zona proviene de esa única empresa.

La unidad de análisis de la investigación fue la empresa. Al ser un caso inédito que, al completarse y documentarse, abrirá la pauta a más casos con condiciones similares de creación de valor, en zonas aisladas en donde la empresa tiene mucho potencial de ser un polo de desarrollo económico para el territorio.

Se debe destacar que, en los ocho casos de Gerentes estudiados por esta empresa, desde el Director Ejecutivo o CEO (Chief Executive Officer por sus siglas en inglés) hasta el Gerente de Recursos Humanos, hubo disposición total a reunirse y ofrecer la complejidad de los datos al 100\%. Entre estos informes o reportes 
de RSE de la empresa, documentos públicos e información de archivo; fotografías e incluso, datos propios y confidenciales.

Para cada uno de los temas en estudio, se consultó y trabajó con el Gerente y el experto de la empresa para validar los datos. Desde esa perspectiva, el caso es exitoso porque existió total disponibilidad de información de parte de la empresa y en cuanto al tiempo de sus Gerentes $^{2}$ para las entrevistas personales realizadas a cada uno de ellos. Esto aunado a la revisión documental de la información secundaria proporcionada. Mediante esto último se pudo comprobar que es una empresa con estándares de calidad global, que maneja sus estadísticas al día. Este aspecto puede favorecer la investigación de otros casos a futuro, en cuanto a la tipología de la empresa.

\section{Resultados}

Los datos recopilados mediante las entrevistas realizadas a los Gerentes de la empresa fueron concentrados en la Matriz general del modelo de valor compartido. Dicha herramienta crea valor en tres niveles:

\section{Re concebir productos y mercados (cómo} la orientación a necesidades no satisfechas genera ingresos y ganancias incrementales).

\section{Redefinir la productividad en la cadena} de valor (cómo una mejor gestión de las operaciones internas aumenta la productividad y reduce los riesgos).

3. Habilitar el desarrollo del clúster (cómo las condiciones sociales cambiantes fuera de la empresa, desatan nuevas ganancias de crecimiento y productividad).

En este primer nivel (Tabla 1) se pueden ver dos creaciones de valor importantes: la inversión en tres años consecutivos en habilidades y competencias de $\$ 995,000$ en Kaizen, Heijunka y TPM, generan a la empresa un total de \$3.6 millones en valor de negocio. Es importante observar que el ROI es de $261.8 \%$, lo que significa que, por cada dólar invertido en el aspecto evaluado, la organización logra ahorros adicionales de \$2.62 en mejora de defectos, manejo de inventarios y productividad.

Asimismo, la empresa invierte en energía fotovoltaica, en uso del agua, acceso para el agua de la comunidad y reutilización del agua por \$2.8 millones. Esto se ve reflejado en la estabilidad para operar, y en el hecho que la planta no cierra operaciones todo el año planificado, lo cual se estima al menos en \$39 millones. Lo anterior reflejaría un ROI de 12.9 veces la inversión social; es decir, por cada dólar invertido en el uso del agua o en el uso de la energía, la empresa está generando un aproximado de $\$ 12.93$ adicionales a la estabilidad del negocio. Así como a la generación de nuevos mercados gracias a los altos estándares de clientes como: Nike, Patagonia y Lululemon.

El segundo nivel (Tabla 2) invierte $\$ 225,000$ en Comandos de Salvamento, parques y campos de fútbol, Policía Nacional y Cruz Roja. Cada dólar invertido le genera estabilidad y seguridad a la empresa, estimada en $\$ 4.5$ millones; de 
Tabla 1. Matriz de creación de valor en el nivel de re concebir productos y mercados

\begin{tabular}{|c|c|c|}
\hline Acciones & Cantidad & ROI* \\
\hline Total de ahorros (inventario + defectos) negocio & \$3.6 millones & \multirow{3}{*}{$261.8 \%$} \\
\hline Total en condiciones mejoradas a la sociedad (Kaizen + Heijunka + TPM) & $\$ 995,000$ & \\
\hline Total de valor compartido (negocios + sociedad) & $\$ 4.6$ millones & \\
\hline Total (crecimiento de mercado + estabilidad) negocio & $\$ 39.0$ millones & \multirow{3}{*}{$1293 \%$} \\
\hline $\begin{array}{l}\text { Total de condiciones medioambientales (acceso a agua }+ \text { uso del } \\
\text { agua }+ \text { reciclaje del agua }+ \text { inversión fotovoltaica) }\end{array}$ & $\$ 2.8$ millones & \\
\hline Total de valor compartido creado (negocio + medioambiente) & \$41.8 millones & \\
\hline
\end{tabular}

*Nota: Entiéndase como retorno sobre la inversión.

Tabla 2. Matriz de la creación de valor en el nivel de redefinir la productividad en la cadena de valor

\begin{tabular}{|c|c|c|}
\hline Acciones & Cantidad & ROI* \\
\hline Total de ahorros (seguridad) negocio & $\$ 4.50$ millones & \multirow{3}{*}{$1900 \%$} \\
\hline $\begin{array}{c}\text { Total de condiciones mejoradas en la sociedad } \\
\text { (Comandos de Rescate }+ \text { Parques }+ \text { Campos de Fútbol }+ \text { PNC) }\end{array}$ & $\$ 225,000$ & \\
\hline Total de valor compartido creado (negocio + sociedad $)$ & $\$ 4.72$ millones & \\
\hline Total (tasa de rotación + tasa de ausentismo) negocio & $\$ 1.20$ millones & \multirow{3}{*}{$292 \%$} \\
\hline $\begin{array}{l}\text { Total de condiciones mejoradas en la sociedad (clínica empresarial } \\
\qquad+ \text { clínica dental }+ \text { unidades de salud) }\end{array}$ & $\$ 306,000$ & \\
\hline Total de valor compartido creado (negocio + sociedad $)$ & $\$ 1.5$ millones & \\
\hline
\end{tabular}

*Nota: Entiéndase como retorno sobre la inversión.

hecho, esto sería un ROI de 19 veces lo invertido en la sociedad o el ambiente. Estos datos representan muy bien lo planteado en la hipótesis del estudio.

Asimismo, la empresa invierte $\$ 306,000$ en una clínica empresarial, una clínica odontológica y en Unidades de Salud de la zona. Por cada dólar invertido, se le generan disminuciones en la tasa de rotación y ausentismo dentro de la empresa, estimadas en $\$ 1.2$ millones, dando como resultado un ROI de 2.92 veces lo invertido en la sociedad. Nuevamente, estas cifras tienen congruencia con lo planteado en la hipótesis.

En el tercer nivel (Tabla 3) se invierte $\$ 1.76$ millones en diez equipos de fútbol que mantiene la empresa; además subsidia escuelas, cafeterías y parques. Todo esto le ha generado a la empresa y a sus trabajadores $\$ 1.82$ millones que se traducen en: generación de otras empresas PYMES; promoción interna del mercado 
de negocios, brindando productos de calidad a bajo costo dentro de las instalaciones de la empresa a los empleados, así como un incremento histórico de las utilidades netas la empresa (33\%). Esta cifra está muy por encima del desempeño de una empresa promedio en cuanto a los resultados de rentabilidad financiera.

En la tabla 4 se presenta un resumen de todo el valor creado en la empresa.

Como se puede apreciar, el valor compartido creado por la empresa (Tabla 4) supera los \$56 millones como valores indicativos de la ejecutoria del negocio. Sin embargo, se debe destacar que el ratio de $\$ 50.12$ millones de valor negocio / \$6.09 millones de valor social pueden indicar una tendencia en ciertos giros de negocios, en donde este resultado de más de ocho veces el valor social invertido, puede ser el camino para hacer más productiva la actividad empresarial; y al mismo tiempo, desatar la innovación en la sociedad.

\section{Discusión}

Los resultados obtenidos de este caso de estudio son bastante claros, porque en todas las áreas, la cantidad invertida en la sociedad está generando valor de negocio tres veces o más, el valor inicial invertido. Resulta interesante continuar midiendo valores en diferentes giros de negocios; un ejemplo de ello es lo que se elabora en Tecnosol, Nicaragua, como producto de esta investigación; siendo una prueba palpable de la inversión social o ambiental.

En la misma línea de discusión, ¿existe un caso de negocio que pueda guiar a más investigaciones en Latinoamérica y El Salvador sobre el

Tabla 3. Matriz de creación de valor en el nivel de habilitar el desarrollo del clúster

\begin{tabular}{|c|c|c|}
\hline Acciones & Cantidad & ROI* \\
\hline $\begin{array}{c}\text { Total de generación de valor de negocio (PYMES + mercado de } \\
\text { negocios para emprendedores) }\end{array}$ & $\$ 1.82$ millones & \multirow{3}{*}{$3.4 \%$} \\
\hline $\begin{array}{l}\text { Total de condiciones mejoradas a la sociedad (equipos de fútbol }+ \\
\qquad \text { escuelas }+ \text { cafetería }+ \text { parques })\end{array}$ & $\$ 1.76$ millones & \\
\hline Total de valor compartido creado (negocio + sociedad) & $\$ 3.5$ millones & \\
\hline
\end{tabular}

*Nota: Entiéndase como retorno sobre la inversión.

Tabla 4. Matriz general del valor compartido total creado en la empresa*

\begin{tabular}{|c|c|c|c|}
\hline $\begin{array}{c}\text { Total de valor de } \\
\text { negocio creado }(\mathbf{N})\end{array}$ & $\begin{array}{c}\text { Total de valor social } \\
\text { creado }(\mathbf{S})\end{array}$ & $\begin{array}{c}\text { Total del valor } \\
\text { compartido creado } \\
(\mathbf{N}+\mathbf{S})\end{array}$ & $\begin{array}{c}\text { Incremento de } \\
\text { utilidades netas } \\
\text { (Histórico) }\end{array}$ \\
\hline$\$ 50.12$ millones & $\$ 6.09$ millones & $\$ 56.21$ millones & $33 \%$ \\
\hline
\end{tabular}

*Nota: Cifras expresadas en USD. 
valor compartido? La respuesta es sí. Después de terminar este caso seminal, queda claro que el modelo diseñado con la meta Matriz que resume el valor compartido de la teoría de Porter, puede llevarse muy bien al nivel de estudio en mediciones dentro de diferentes países. Y para el caso, con un enfoque en Latinoamérica.

¿Existe alguna relación entre la creación de valor compartido y el retorno de estas inversiones? Si bien existe un estudio sobre retorno de inversión, este aparentemente centra su línea de acción en cuanto a que, salvando muchos problemas sociales, se puede generar riqueza impresionante y un crecimiento acelerado en las empresas. Esto es totalmente compatible con el discurso de Porter. Cuando se invierte en los grandes problemas sociales como: falta de formación y habilidades en las personas; acceso a agua potable, energía, educación, seguridad y salud, es cuando el dinero invertido se traduce en valor de negocio, en un ratio de 12 a 18 veces el valor de la inversión.

El enfoque regional de este caso se presentó en diciembre de 2019 frente al concejo de Impacto Global de la Escuela de Negocios de Harvard; además de contar con la ayuda de los profesores: Felipe Pérez de INCAE Businnes School; Alfonso Mendoza y Pablo Nuño de la Universidad Popular Autónoma del Estado de Puebla (UPAEP), México.

\section{Referencias}

Ballantine, H.W. (1932). The Modern Corporation and Private Property. California Law Review, $21(1) ; 78$.

Berle, A. (1931). Corporate powers as powers in trust, Harvard Law Review, 44; 1049-1076.

Drucker, P.F. (1984). Converting social problems into business opportunities: The new meaning of corporate social responsibility, California Management Review, 26 (2); 53-63.

Freeman, R.E. (1984). Strategic management: A stakeholder approach. Pitman Boston.

Friedman, M. (1970). The social responsibility of business is to increase its profits. New York Times Magazine, 13; 32-33.

Hart, S.L. (1995). A Natural-Resource-Based View of the Firm. Academy of Management Review, 20 (4), 986-1014.

Hart, S.L. (1997). Beyond Greening: Strategies for a Sustainable World. Harvard Business Review, 75 (1), 66-76.

Hart, S.L. (2005). Capitalism at the crossroads: The unlimited business opportunities in solving the world's most difficult problems. Pearson Prentice Hall. 
Hart, S.L. (2007). Capitalism at the crossroads: Aligning business, earth and humanity. Wharton School Pub.

Hart, S.L. (2010). Capitalism at the crossroads: next generation business strategies for a post-crisis world. Wharton School Pub.

Hart, S.L. y Dowell, G. (2011). Invited Editorial: A Natural-Resource-Based View of the Firm: Fifteen Years After. Journal of Management, 37 (5); 1464-1479.

Hediger, W. (2010). Welfare and Capital-Theoretic Foundations of Corporate Social Responsibility and Corporate Sustainability. Journal of Socio-economics, 39 (4); 518-526.

Holme, R. y Watts, P. (2000). Corporate social responsibility: making good business sense, World Business Council for Sustainable Development.

Jamali, D. y Mirshak, R. (2007). Corporate social responsibility (CSR): theory and practice in a developing country context, Journal of Business Ethics, 72 (3); 243-262.

McElhaney, K. (2009). A strategic approach to corporate social responsibility. Leader to Leader, $52(1) ; 30-36$.

McWilliams, A. y Siegel, D. (2001). Corporate social responsibility: A theory of the firm perspective. Academy of Management Review, 26 (1); 117-127.

McWilliams, A.; Siegel, D.S. y Wright, P.M. (2006). Corporate social responsibility: Strategic implications. Journal of Management Studies, 43 (1); 1-18.

Porter, M.E. y Kramer, M.R. (2002). The competitive advantage of corporate philanthropy, Harvard Business Review, 80 (12); 56-68.

Porter, M.E. y Kramer, M.R. (2006). The link between competitive advantage and corporate social responsibility, Harvard Business Review, 11; 1-13.

Porter, M. E.; Kramer, M. R. y Lorsch, J. W. (2009). Ser Competitivo. Barcelona, España: Deusto.

Porter M. E. y Kramer M. (2011, January-February). The big idea, Creating Shared Value. Harvard Business Review; 1-17.

Prahalad, C. K. y Hart, S. L. (2002). The Fortune at the Bottom of the Pyramid. Strategy+ Business. Booz Allen Hamilton.

Russo, M.V. y Fouts, A. F. (1997). A Resource-Based Perspective on Corporate Environmental Performance and Profitability. Academy of Management Journal, 40 (3); 534-

Salamon, L.M. (1992). America's nonprofit sector. Washington, DC.: Foundation Center 
Samuelson, P.A. (1971). Generalized predator-prey oscillations in ecological and economic equilibrium. Proceedings of the National Academy of Sciences, 68 (5); 980-983.

Umana, M. (2013). The Function and the Equation of Sustainability. A dissemination of a new advancement in the evolution of sustainable development. Recuperado de: https://www-csd. eng.cam.ac.uk/proceedings-of-the-eesd13-conference-cambridge-2013-v-2/eesd13-published-papers/umana-m.pdf/view

Umaña, M. V. (2019). Capital semilla y la ecuación de inversión en emprendimientos. Anuario de Investigación, 8; 59-71.

Vidal, I. (2011). El principio de valor compartido de Porter y Kramer. Foment del Treball Nacional (Fomento del trabajo), (2134); 30-33.

Wang, H.; Choi, J. y Li, J. (2008). Too little or too much? Untangling the relationship between corporate philanthropy and firm financial performance. Organization Science

Zainal, Z. (2007). Case study as a research method. Journal Kemanusiaan, 5 (1). 